\title{
A NOTE ON SUPPORT POINTS
}

\author{
BY N. T. PECK
}

Communicated by Ernest Michael, May 27, 1968

Bishop and Phelps proved in [1] that every proper closed convex subset of a Banach space has "many" support points. (A support point of a convex set $C$ in a real topological linear space $E$ is a point $x$ of $C$ for which there is a nonzero continuous linear functional $f$ on $E$ with $f(x)=\sup _{c \in C} f(c)$.) At the end of their paper they asked whether "Banach space" can be replaced by "complete locally convex space" in the statement of this result. In [3], Klee settled this negatively by exhibiting a proper closed convex set in $R^{\aleph_{0}}$ with no support points. The set in Klee's example is unbounded, and at the end of [3] the question was raised whether a bounded closed convex subset of a complete locally convex space must have support points. (Note that a bounded closed convex subset of $R^{k_{0}}$ is weakly compact and hence has support points. Indeed, if $\left\{B_{i}\right\}_{i=1}^{\infty}$ is any sequence of reflexive Banach spaces, every bounded closed convex subset of the product space $\prod_{i=1}^{\infty} B_{i}$ is weakly compact and therefore has support points.)

We settle Klee's question negatively:

THEOREM. Let $\left\{B_{i}\right\}_{i=1}^{\infty}$ be any sequence of nonreflexive Banach spaces. Then in the product space $\prod_{i=1}^{\infty} B_{i}$ there is a closed bounded convex set which has no support points.

The construction of the example rests on the theorem of James [2], that on every nonreflexive Banach space $B$, there is a continuous linear functional which does not assume its supremum on the unit ball of $B$. The detailed proof and related results will appear elsewhere.

\section{REFERENCES}

1. Errett Bishop and R. R. Phelps, The support functionals of a convex set, Proc. Sympos. Pure Math., vol. 7, Amer. Math. Soc., Providence, R. I., 1963, pp. 27-35.

2. R. C. James, Weakly compact sets, Trans. Amer. Math. Soc. 13 (1964), 129-140.

3. V. L. Klee, On a question of Bishop and Phelps, Amer. J. Math. 85 (1963), 95-98.

The University of Warwick, Coventry, England 